\title{
Matrix Metalloproteinases and Their Inhibitors in Kidney Scarring: Culprits or Innocents
}

\author{
Aimun Kamal Hussein Ahmed* \\ The Sheffield Kidney Institute, Northern General Hospital, Sheffield Teaching Hospitals NHS Foundation Trust, Sheffield, United King- \\ dom
}

(Received September 20, 2008; Accepted May 18, 2009)

\begin{abstract}
The renal extracellular matrix (ECM) is in a continual state of turnover with homeostasis maintained by balancing synthesis and degradation rates. During progressive kidney scarring an imbalance occurs leading to increase ECM either by increased deposition or decreased breakdown, although a combination of the two is more likely. Increased synthesis of many ECM proteins such as collagens I, III, and IV, fibronectin and laminin contribute to this imbalance; however decreased proteolytic activity leading to accumulation of ECM components is also an important component of scarring. The matrix metalloproteinases (MMPs) system is predominantly responsible for degrading mature ECM, consisting of 24 members, with MMP's 1, 2, 3, 8, 9, and 13 having significant renal expression. The kidney also expresses 3 Tissue inhibitors of MMPs; TIMP-1, 2, and 3. MMPs also have a role during kidney development. Several studies describe changes in ECM proteolysis and more specifically MMPs in end stage kidney disease (ESRD), although most of these are descriptive and based on enzymatic, protein or mRNA analysis of homogenates. There is no consistency in most of these studies regarding the expression of MMPs and TIMPS in experimental kidney scarring. Few of these studies have been investigated in human kidney scarring. Here is a comprehensive review of the MMPs and TIMPs literature including their action, activation, regulation, and contribution in experimental and human CKD.
\end{abstract}

Key words — matrix metalloproteinases, tissue inhibitors of matrix metalloproteinases, chronic kidney disease, kidney scarring, kidney fibrosis

\section{INTRODUCTION}

Kidney scarring is an inevitable consequence of progressive chronic kidney disease (CKD). Kidney scarring leads to end stage renal disease (ESRD), a devastating disorder which has a common histological end point to most progressive kidney diseases, such as diabetes mellitus, glomerulonephritis, focal sclerosis, chronic transplant dysfunction, etc. ${ }^{1)}$ Kidney scarring represents a failed woundhealing process of the kidney tissue after chronic, sustained injury, with loss of resident kidney cells and their replacement by infiltrating inflammatory cells and fibrous tissue. Several cellular pathways, including mesangial and fibroblast activation

\footnotetext{
*To whom correspondence should be addressed: The Sheffield Kidney Institute, Northern General Hospital, Sheffield Teaching Hospitals NHS Foundation Trust, Sheffield, United Kingdom. Tel.: +0114-271-4663; Fax: +0114-256-2514; E-mail: aimun@doctors.org.uk
}

as well as tubular epithelial-mesenchymal transition (EMT) have been involved in the generation of the matrix-producing cells leading to extracellular matrix (ECM) accumulation. ECM is in continuous state of turnover with a tight balance between its synthesis and degradation, during progressive kidney scarring there is imbalance with either increased deposition or decreased breakdown or a combination of both. ${ }^{2,3)}$ The mechanisms behind the accumulation of ECM in progressive CKD are important from both a pathogenic perspectives, and as potential targets for therapeutic intervention. ${ }^{4)}$ Many fibrogenic factors regulate kidney fibrotic process, as well as matrix-degrading enzymes. Interstitial recruitment of inflammatory leukocytes and myofibroblasts occurs early in kidneys developing fibrosis. Circulating monocytes are recruited by locally secreted chemoattractant molecules, facilitated by leukocyte adhesion molecules. ${ }^{5)}$ The matrix metalloproteinases (MMPs) system is considered one of the major systems responsible for degrading accu-

(C)2009 The Pharmaceutical Society of Japan 
mulated ECM and balancing the ECM turnover., ${ }^{3,6)}$ Here is a comprehensive review of their action, activation, regulation, and their contribution in experimental and human CKD.

\section{THE KIDNEY ECM}

The ECM in the kidney like else where in the body, a three-dimensional network of macromolecules (collagens, elastin, fibronectin, laminins and glycosaminoglycans). ${ }^{7)}$ ECM plays an integral role in basement membrane composition, cell development, homeostasis, physiology and pathology. It functions through interaction with specific receptors that regulate many aspects of cellular behavior including growth, migration, differentiation, repair, and serves not only as a structural scaffold but also as an instructive environment for tissues and cells. The ECM transmits signals from cells to ECM and vice versa, mediating cell adhesion, proliferation, and survival. Basement membranes (BM) are important components formed of thin sheets of specialized ECM, which support epithelial cell layers. The main components of the kidney ECM are collagen types I, III, IV, and V. Other abundant ECM proteins include laminin, fibronectin, heparan sulphate proteoglycans (HSPG), and chondrotinin sulphate proteoglycan. Type $\mathrm{V}$ is a fibrillar collagen and is found mainly in the mesangium and in the interstitium. The glomerulus has three distinct ECMs, although they all have BM constituents as their major matrix types. The Bowman's capsule has a double BM matrix, while the mesangial cells are surrounded by another pericellular matrix. The third matrix is that of capillary loop. These highly specialised matrices are composed of type IV collagen, laminin, HSPG, fibronectin and chondroitin sulphate proteoglycan. ${ }^{8,9)}$ The composition of each matrix helps to determine the physical, mechanical, and functional properties of the glomerulus. Laminin is involved in cell attachment within the glomerular BM (GBM). Heparan sulphate proteoglycans is essential component of the GBM maintaining negative charge necessary to prevent negative charged molecule filtration, which means prevention of loss of protein in urine. ${ }^{10)}$ The two most abundant mesangial structural proteins are type IV collagen and laminin. It is different from the interstitium ECM having less collagen type I and III. However mesangial cells can produce collagen types I, III, IV, fibronectin, and laminin and other components of the mesangial matrix. Moreover, it is likely that the mesangial cells also regulate the turnover of the constituent components of the matrix. Six different types of collagen IV $\alpha$ chains have been identified in the glomerulus. Glomerular ECM components are synthesized mainly by the podocytes, endothelial cells, resident fibroblasts and to lesser extent by mesangial cells. Glomerular epithelial cells can also produce collagen types I, III, and IV, fibronectin, and laminin, while glomerular endothelial cells produce collagen type IV. ${ }^{8)}$ The kidney interstitial ECM consists of the tubular basement membrane (collagen IV, laminin, and nidogen). While the interstitial proteins is composed mainly of collagens type I, III, V, VII, XV, fibronectin, and proteoglycans, produced by tubular epithelial cells and interstitial fibroblasts. ${ }^{2)}$ Most of the kidney ECM components (collagens, fibronectins, laminins, elastins, etc.) are synthesised intra-cellularly and passed extracellularly in various stages of completion to be deposited into the matrix. However it has been shown that kidney cells like mesangial cells and fibroblasts in cultures are capable of producing ECM components which they would not normally produce. ${ }^{8}$ Subsequently the production of type I and III collagens by the mesangial cells in culture suggests they could be the source of interstitial collagens seen within the glomerulus in progressive kidney scarring. ${ }^{10)} \mathrm{Re}$ modelling of the kidney ECM is an important physiological feature of normal growth and development. There is a tight balance between kidney ECM synthesis and degradation. Changes in either of these dynamic parameters will potentially result in ECM deposition and accumulation within the glomerulus and the tubulo-interstitium compartments. ${ }^{11)}$ It is generally believed that excessive ECM deposition seen in kidney scarring is the result of both over production of ECM components and the lack of their clearance. $^{2)}$

\section{THE MMPs}

MMPs, is a group of zinc endopeptidases secreted by a variety of cell types including the kidney cells, MMPs play a central role in ECM breakdown in various kidney diseases. MMPs activity is tightly controlled by a specific class of natural inhibitors known as tissue inhibitors of metalloproteinases (TIMPs). MMPs, is a big family of 28 members (MMP1-28) involved in remodeling of 


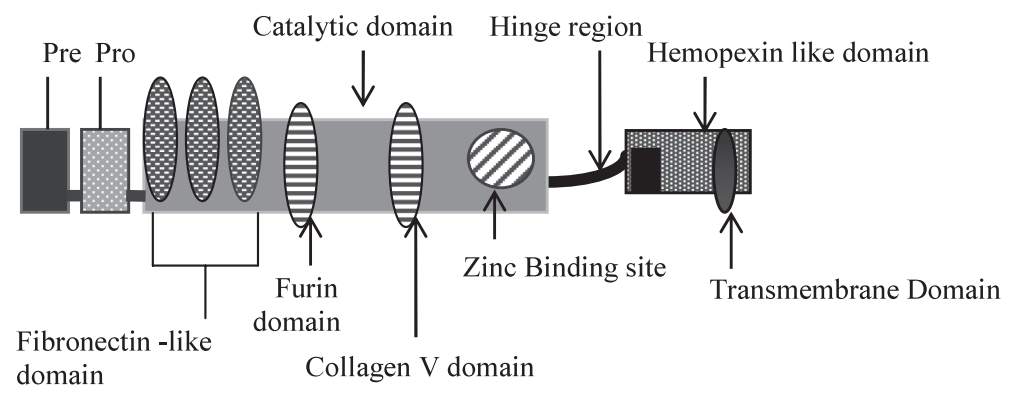

Fig. 1. General MMP Structure

MMPs have a leader sequence, propeptide domain, and a catalytic domain with the zinc-binding site (Zn). The majority of family members have a hinge and hemopexin-like domain. Other domains are represented in one or more family members, such as the fibronectin-like domain, collagen type $\mathrm{V}$-like domain, and transmembrane domain.

ECM through degrading and turning over all of its components. MMPs have been viewed as bulldozers, destroying ECM and promoting tissues remodeling in health and tissue destruction in pathological diseases. MMPs are zinc and calcium-dependent enzymes that are tightly regulated and expressed constitutively at low levels in most cell types and tissues. Their expression can be induced and up regulated during active normal and abnormal tissue remodeling processes, such as ebryonic development, tissue repair, inflammation, tumour invasion and metastasis. Each MMP member has the potential to degrade at least one component of the ECM, with maximal activity around neutral $\mathrm{pH} .{ }^{12)}$ MMPs have been classified based on their substrate specificity into different subclasses which include the interstitial collagenases (MMP-1, MMP-8, MMP-13), stromelysins (MMP-3, MMP-7, MMP-10, MMP11), gelatinases (MMP-2, and MMP-9), and membrane type MMP (MT-MMP) (MMP-14, MMP-15, MMP-16, MMP-17, MMP24, and MMP-25) (Table 1). ${ }^{3,13)}$ Most MMPs are secreted; however The MT-MMPs display transmembrane domains and are expressed on the cell surfaces. MMPs are multidomain enzymes and they are grouped according to their domain structure as is shown in Fig. 1. All MMPs are synthesized as inactive preproenzymes. The "pre" domain is an $N$-terminal signal sequence which directs MMPs synthesis to the endoplasmic reticulum and gets removed as the proenzyme is secreted from the cell. The pro-domain also serves to keep the enzyme minimally active until its activity is required, by serving as internal MMPs inhibitor. Cleavage of the pro-domain leads to a conformational change in MMPs and they become active. ${ }^{14)}$ Their central catalytic domain contains amino acid residues that bind a central zinc ion and 2-3 cal- cium ions, both are essential for stability and activation. The purpose of the propeptide cleavage is to expose this zinc ion which is essential for MMPs activation, and explains the word 'metallo' in their name. Most of the MMPs except MMP7, and MMP-26, have a disulfide-bonded carboxyterminal portion (hemopexin domain region) which is connected to the catalytic domain by a hinge, and is thought to mediate additional protein-protein interactions with substrates (substrate specificity), and interaction with TIMPs. ${ }^{15)}$ The hinge region, in turn, varies in length and composition among the various MMPs and also influences substrate specificity. ${ }^{16,17)}$ Gelatinases (MMP-2 and MMP-9) are characterized by having three cysteine-rich repeats within their catalytic domain, which are believed to be fibronectin-type II like domain. These inserts are required to bind and cleave collagens. ${ }^{14)}$ Some MMPs like MMP-9 has a type V collagen ( $\mathrm{Col} \mathrm{V}$ ) domain which has a role in modulating protein interactions important in substrate recognition. The MTMMPs with the exception of MMP-17 and MMP25 , have a single-pass transmembrane domain and a short cytoplasmic C-terminal tail (about 20 amino acids). The cytoplasmic domain of MT-MMPs may have a role in the regulation of their activity on the cell surface. On the other hand MT-MMP-17 and MT-MMP-25 have a glycosyl-phosphatidyl inositol (GPI)-anchored domains, which are important in cell surface localization and activation. ${ }^{18)}$ Other domains like the furin domain helps in providing an alternative cleavage site for MMP activation. Ten members of MMPs are expressed in the kidney; MMP-1, -2, -3, -9, -13, -14, -24, -25, -27, -28, and six members have been extensively studied in the kidney; MMP-1, -2, -3, -9, -13, and MT-1-MMP. ${ }^{19)}$ 
Table 1. The MMPs

\begin{tabular}{|c|c|c|c|}
\hline Enzymes & MMP number & Matrix substrates & Producing kidney cells \\
\hline \multirow[t]{5}{*}{ Collagenases } & MMP-1 (55 kD) & $\begin{array}{l}\text { Collagens types I, II, III, VII, VIII, X, } \\
\text { gelatin, and proteoglycan, (degrading } \\
\text { collagen III }>\text { I) }\end{array}$ & $\begin{array}{l}\text { Mesangial cell, endothelial cell, } \\
\text { tubulo-epithelial cell, fibroblasts, } \\
\text { macrophage }\end{array}$ \\
\hline & MMP-8 (75 kD) & Collagens types I, II, III (degrading & Neutrophils \\
\hline & Collagenase-2 & collagen type I $>$ III & \\
\hline & MMP-13 (65 kD) & Collagen types I, II, III & Fibroblasts \\
\hline & Collagenase-3 & & \\
\hline \multirow[t]{4}{*}{ Gelatinases } & MMP-2 (72 kD) & Collagens types III, IV, V, VII, X, & Mesangial, glomerular, epithe- \\
\hline & Gelatinase A & $\begin{array}{l}\text { gelatin, fibronectin, laminin, } \\
\text { proteoglycans and elastin }\end{array}$ & $\begin{array}{l}\text { lial, endothelial cells, and tubu- } \\
\text { lar cells (collecting ducts), fibrob- } \\
\text { lasts, macrophages. }\end{array}$ \\
\hline & MMP-9 (92 kD) & Collagen types III, IV,V, gelatin, & Same as MMP-2 \\
\hline & Gelatinase B & proteoglycans, elastin, and entactin & \\
\hline \multirow[t]{5}{*}{ Stromelysins } & MMP-3 (57 kD) & Collagen types IV, VII, IX, X, elastin, & Mesangial, tubulo-epithelial cells, \\
\hline & Stromelysin-1 & proteoglycan, gelatin, fibronectin, lamnin. & fibroblasts. \\
\hline & MMP-10 (58 kD) & Collagen types, IV, V, proteoglycan, & Fibroblasts \\
\hline & Stromelysin-2 & fibronectin, and laminin & \\
\hline & $\begin{array}{l}\text { MMP-11 (51 kD) } \\
\text { Stromelysin-3 }\end{array}$ & $\begin{array}{l}\text { Collagen types IV, fibronectin, laminin, } \\
\text { gelatin, and elastin. }\end{array}$ & Unkown \\
\hline $\begin{array}{l}\text { Matrilysin } \\
\text { Pump-1 }\end{array}$ & MMP-7 (28 kD) & $\begin{array}{l}\text { Proteoglycan, fibronectin, gelatin, and } \\
\text { entactin }\end{array}$ & $\begin{array}{l}\text { Mesangial cells, uterine cells, } \\
\text { macrophages }\end{array}$ \\
\hline \multirow{12}{*}{$\begin{array}{l}\text { Membrane- } \\
\text { type MMPs }\end{array}$} & MMP-14 (63 kD) & Collagen types I, II, III, gelatin, & Mesangial, and fibroblasts \\
\hline & MT1-MMP & $\begin{array}{l}\text { fibronectin, laminin, proteoglycan, } \\
\text { collagen I, II, III, ProMMP2, ProMMP13 }\end{array}$ & \\
\hline & MMP-15 (72 kD) & Gelatin, fibronectin, tenascin, nidogen, & Unkown \\
\hline & MT2-MMP & aggrican, percan. & \\
\hline & MMP-16 (64 kD) & Collagen III, gelatin, fibronectin, & Unkown \\
\hline & MT3-MMP & ProMMP2 & \\
\hline & MMP-17 & Gelatin, ProMMP2 & Unkown \\
\hline & MT4-MMP & & \\
\hline & MMP-24 (53 kD) & Elastin, fibronectin, gelatin, ProMMP2 & Unkown \\
\hline & MT5-MMP & & \\
\hline & MMP-25 (63 kD) & Gelatin, ProMMP2 & Unkown \\
\hline & MT6-MMP & & \\
\hline
\end{tabular}

\section{MMPs ACTIVATION AND REGULATION}

Activation of MMPs is mainly occurring in the pericellular environment away from natural inhibitors. The activation process occurs by use of a mechanism called the cysteine switch. MMPs are folded by the binding of amino acid 73 (cysteine) via a disulphide bond to the zinc molecule in the active site thus blocking the catalytic site. ${ }^{19)}$ To activate the switch a water molecule must be allowed access to the zinc molecule, leading to dissociation of the thiol-bearing propeptide from the active zinc atom. Once the link between the cysteine and zinc is broken the protein undergoes a conformational change and the MMPs undergo auto proteolysis to release the propeptide and gain full activity. The opposite happens when cysteine is "on" the zinc, the activity of the enzyme is "off." Thus, the dissociation of cysteine from the zinc atom is viewed as the "switch" that leads to activation. It is believed that such activation (The cysteine-switch mechanism) occurs in the pericellular and extracellular environment, and is applicable to all members of MMPs. There are other pathways of in vivo activation where MMPs are activated by other active MMPs, like the extra cellular activation of proMMP-9 by active MMP-2, MMP-3 and MMP-13. proMMP-13 also can be activated by active MMP- 2 and MMP3, also MMP-3 activates proMMP-1. ${ }^{20)}$ The membrane type-MMPs can also activate secreted MMPs like the activation of proMMP-2 by MT1-MMP in a pathway which is TIMP-2 dependent. ${ }^{20)}$ However MT2-MMP activates proMMP-2 in a pathway that is TIMP-2 independent, but MMP-2 hemopexin car- 
Table 2. TIMPs

\begin{tabular}{|c|c|c|}
\hline TIMPs & Inhibitory effect & Producing kidney cells \\
\hline TIMP-1 (28 kD) & $\begin{array}{l}\text { All the latent proMMPs and active MMPs. } \\
\text { Does not inhibit MMP-14, }-16 \text {, or }-24 \text {. } \\
\text { Poor inhibitor of MMP-19 and a number of MT-MMPs }\end{array}$ & $\begin{array}{l}\text { All mesenchymal tissues including } \\
\text { intrinsic glomerular cells (mesan- } \\
\text { gial cells and capillary endothelium), } \\
\text { macrophages, and fibroblasts. }\end{array}$ \\
\hline TIMP-2 (23 kD) & All the latent proMMPs and active MMPs. & Mesangial, glomerular epithelial cells \\
\hline TIMP-3 (21 kD) & $\begin{array}{l}\text { All the latent proMMPs and active MMPs. } \\
\text { Inhibits A Disintegrin and a Metalloproteinase } \\
\text { (ADAMs), as well as the matrix-associated ADAM } \\
\text { with a thrombospondin-like motif (ADAM-TS) }\end{array}$ & Fibroblasts \\
\hline TIMP-4 (22 kD) & MMP-1, MMP-2, MMP-3, MMP-7, and MMP-9 & Unknown \\
\hline
\end{tabular}

boxyl (C) domain dependent. ${ }^{21)}$ MT1-MMP also activates proMMP-13.

MMPs are not available at high levels in the kidney ECM and their expression is tightly regulated by growth factors, cytokines, chemical agents (e.g., phorbol esters), and physical stress. They can be down regulated by suppressive factors [e.g., transforming growth factor-beta (TGF- $\beta_{1}$ ), retinoic acids, glucocorticoids, interleukin (IL-1)]. MMPs are also tightly regulated by hormones, tumour promoting agent PMA, and oncogene products. ${ }^{22)}$ MMPs can be induced by [IL- $1 \beta$, tumour necrosis factor-alpha (TNF- $\propto$ ), TGF- $\propto$, epidermal growth factor (EGF), fibroblast growth factor (FGF), PMA, phorbol ester ${ }^{23)}$ and platelet-derived growth factor (PDGF)], and repressed by (TGF- $\beta_{1}$ and IL-4) see Table $3 .^{24,25)}$ TGF- $\beta_{1}$ was shown to reduce interstitial collagenase mRNA, decreases the interstitial collagenase on protein level and increases the protein level of TIMP-1, -2 and $-4{ }^{26)}$ TGF- $\beta_{1}$ exerts a striking reciprocal effect on the balance between MMPs and TIMPs expression; TIMPs expression and secretion is synergistically enhanced, and collagenase expression selectively inhibited. These reciprocal effects of TGF- $\beta_{1}$ on opposing activities effectively amplify the inhibitory arm of the MMPs/TIMPs system leading to a net inhibition of extracellular matrix breakdown. ${ }^{16)}$ However exceptionally TGF- $\beta_{1}$ enhances MMP-2 in cultured mesangial cells. ${ }^{27)}$

\section{TIMPs}

TIMPs are multifunctional proteins and the major endogenous regulators of MMPs activities in the tissue. Four distinct TIMPs molecules (21$30 \mathrm{kDa}$, TIMPs-1 to 4) have been isolated, cloned, sequenced, and characterised from several species
Table 3. Growth Factors and Cytokines Inducing (+) or Inhibiting (-) MMPs and TIMPs

\begin{tabular}{lll}
\hline \hline & \multicolumn{1}{c}{ MMPs } & \multicolumn{1}{c}{ TIMPs } \\
\hline IL-1beta & + MMP-1, 2, and 3 & + TIMP-1, and -2 \\
IL-1alpha & + MMP-1, 2, and 3, & +TIMP-1, -2, and -3 \\
& + +MMP1, 3, 7 & +TIMP-1 \\
TNF- $\propto$ & + MMP & + TIMP-1 \\
TGF-beta1 & + MMP2 & + TIMP-1, and -3 \\
& - most MMPs & + +mRNA TIMP-1 \\
& + mRNA MMP2 & - TIMP-2 \\
PDGF & + MMP & + TIMP \\
IL-6 & & + TIMP \\
tPA & + MMP & + TIMP \\
FGF-2 & + MMP & + TIMP-1, and -3 \\
EGF & + MMP & + TIMP-1, and -3 \\
Retinoids & - MMP & + TIMP \\
\hline
\end{tabular}

tPA: Tissue type plasminogen activator.

(Table 2). ${ }^{28)}$ All TIMPs inhibit active MMPs with relatively low selectivity, forming tight noncovalent 1:1 complexes with the activated catalytic zinc in the MMPs. Details of the MMP-TIMP interaction were a mystery for years until the crystal structure of the complex formed between TIMP1 and the catalytic domain of MMP-3 was discovered by Gomis-Rüth et al. (1997). ${ }^{29)}$ Most of the intermolecular contacts between these two proteins are restricted to the first five amino-terminal residues of TIMP-1, which binds to the active site in a substrate-like manner. Tissue inhibitors of metalloproteinases are not actually changed or modified by their interaction. The concept exists that a balance between the level of active MMPs and their inhibitors regulates proteolytic activity. The TIMPs have six disulphide bonds and comprise a three-loop N-terminal domain and an interacting three-loop C-subdomain. The TIMPs are secreted proteins, but may be found at the cell surface in association with MT-MMPs; for example, 
TIMP-2, TIMP-3 and TIMP-4 could bind MMP-14. Uniquely, TIMP-3 is sequestered to the ECM by binding to heparan-sulphate-containing proteoglycans and possibly chondroitin-sulphate-containing proteoglycans. ${ }^{30)}$

\section{MMPS AND THEIR INHIBITORS IN THE DEVELOPING KIDNEY}

Kidney development is characterized by a high rate of ECM turnover. During renal development, a constant remodeling of ECM is required to allow invasion and branching of the ureteric bud in the metanephric mesenchyme. ${ }^{31)}$ MMPs play an important role in epithelium-mesenchyme interactions in renal development and the determination of nephron number in the developing kidney. MMP-2 mRNA expression is limited to the mesenchyme. However, MMP-2 protein has been found in immature nephron structures undergoing epithelial differentiation, where it co-localizes with MT1-MMP, TIMP-2, and TIMP-3. ${ }^{32}$ ) This has given rise to the hypothesis that the interaction between MMP-2, MT-1-MMP, and TIMP-2 may be crucial for the interaction between mesenchyme and epithelium, facilitating branching of the developing ureteric bud. Inhibition of MMP-2 by TIMP2 reduces significantly the number of branches in the rat metanephros. ${ }^{33)}$ Branching morphogenesis of the ureteric bud requires functional MMP9 activity, anti-MMP-9 antibody preventing ureteric bud branching. ${ }^{34)}$ Also other MMPs, such as MMP-1, have been shown to play a role in branching morphogenesis. ${ }^{35)}$

\section{MMPS AND THEIR INHIBITORS IN EXPERIMENTAL CKD}

Involvement of the MMPs/TIMPs system has been implicated in kidney tubulo-interstitial fibrosis and glomerulosclerosis for more than two decades. The imbalance in the MMPs/TIMPs system with reduced MMPs activity, and the up regulation of the natural inhibitors TIMPs was very popular theory, where extensive ECM accumulation leads to progression of kidney scarring. ${ }^{36,37)}$ Many studies have measured MMPs and or their activity in experimental models of inflammatory and non inflammatory glomerular disease, and in progressive kidney scarring. Down regulation of MMPs has been associated with progression in hypertensive glomerulosclerosis, ${ }^{38)}$ heroin nephropathy, ${ }^{39)}$ sex-related changes in the aging kidney, ${ }^{40)}$ Unilateral Ureteric Obstruction (UUO), ${ }^{41)}$ and renal ligation. ${ }^{42)}$ In the UUO rat model it was shown that both the gelatinolytic and collagenolytic activity were reduced in the evolution of interstitial kidney fibrosis, and that was mainly due to increase TIMP-1 protein and lack of activation of latent forms of MMP-1 and MMP-9. ${ }^{41)}$ Johnson et al. $(2002)^{36)}$ previously measured the MMPs activity in the 5/6 subtotal nephrectomized (SNx) rat, a model of CKD which develops proteinuria, hypertension, and increased matrix protein deposition leading to decline in kidney function. Johnson and colleagues reported raised TIMP1 and TIMP-3 mRNA at day 90 post SNx in comparison to normal control. The overall collagenase activity was reduced in the SNx homogenate using collagen I and IV substrates. However, there was increase in mRNA for MMP-1 and MMP-2, indicating an inhibitory effect by TIMPs rather than reduced MMPs transcription or activation. Most of the studies which measured ECM activity were homogenate based assay and conclusions were drawn on an overall activity rather than in situ localized activity or immunohistochemical localization of MMPs or TIMPs members. Others have shown various different expressions of TIMPs/MMPs members in various experimental kidney models. MMP1 immunostaining was shown to be upregulated in the cytoplasm of the tubular epithelial cells in the cisplatin nephropathy rat model of kidney scarring. However late as the scarring progressed and collagen III became deposited there was decreased in MMP-1 levels intracellularly. ${ }^{43)}$ In the anti-Thy 1.1 nephritis, rat model of mesangial proliferative glomerulonephritis, an increase in proliferation of mesangial cells is associated with massive expression of MMP-2 and accumulation of extracellular matrix proteins, and that was observed within foci of proliferating mesangial cells and injured basement membrane. These inflammatory features were shown to be attenuated by a synthetic MMPs inhibitor. ${ }^{44)}$ It was shown that TGF- $\beta_{1}$ has a role in inducing MMP-2 in the same model and in other models of induced nephritis where both MMP-2 and TGF- $\beta_{1}$ mRNA were elevated. ${ }^{45,46)}$ MMP-2 mRNA was also increased initially then fell progressively in renal ischaemia-reperfusion injury rat model in association with increase mRNA of TIMP1, and TGF- $\beta_{1}{ }^{47)}$ In the rat streptozotoci model of diabetic nephropathy it was described 
that MMP-2 immunostain decreased intracellular in the glomeruli (epithelial and mesangial cells). ${ }^{48)}$ Also in the glomerlonephritis-hereditary nephritic mice, lower activity levels of MMP-1, -2, and -9 but not MMP-3 was shown. ${ }^{49)}$ In another model of experimental scarring induced by nephrectomy, MMP-1, -3 mRNA levels were shown to increase 3 folds within the kidney cortex $24 \mathrm{hr}$ following unilateral nephrectomy, and then gradually decreased to the control level after 7 days. In contrast the expression of MMP-2 and MMP-9 which remained unchanged in the same model, in association with significant increase in TIMP-1 mRNA levels within the renal cortex $12 \mathrm{hr}$ following nephrectomy. ${ }^{50)}$ MMP-9 was shown to decrease by immunhistochemistry during progressive glomerulosclerosis in the obese Zucker rat model of insulin resistance and hyperlipidaemia in association, there was decreased gelatinolytic activity with upregulation of TIMP1 protein levels. ${ }^{51)}$ MMP-9 has been up regulated in the glomeruli of proteinuric rat model with passive Heymann nephritis, ${ }^{52)}$ also MMP-9 immunhistochemical staining was significantly higher in the cytoplasm of tubular cells of the acute pyelonephritis model of renal scarring. ${ }^{53)}$ TIMP-1 was thought to be a key player in ECM deposition associated with CKD, TIMP-1 was found to be significantly elevated in several experimental models of kidney scarring like, protein-overload proteinuria rat model ${ }^{54)}$ hypercholesterolaemia-induced renal disease, ${ }^{55)}$ obstructive uropathy ${ }^{56,57)}$ anti tubular basement membrane antibody nephritis, ${ }^{58)}$ murine lupus nephritis, ${ }^{59)}$ polycystic kidney disease, ${ }^{60,61)} \mathrm{cy-}$ closporine nephrotoxicity, ${ }^{62)}$ Heymann nephritis, ${ }^{52)}$ and the acute pyelonephritis model of kidney scarring. ${ }^{53)}$ However, the importance of TIMP-1 was questioned by Eddy and colleagues ${ }^{63)}$ who showed in an experimental model of bovine serum albumin (BSA)-induced overload proteinuria in TIMP1 knockout mice that genetic deficiency of TIMP1 does not appear to protect from developing severe interstitial fibrosis, but that was explained by the high renal constitutive levels of TIMP-2 and/or TIMP-3. We have previously shown MMPs activity in the remnant kidney model to drop significantly in progressive kidney scarring. Using in situ zymograpghy, were able to localize MMPs activity within the tubules in normal rat kidney which was inhibited by adding MMPs specific inhibitor. MMPs activity dropped significantly in the tubules in progressive scarring associated with increase in tubular TIMP-2 by immunohistochem- istry. The tubulo-interstitial forms the main bulk of the kidney mass, therefore the overall activity was reduced accordingly. However there was unexpected increase in the glomerular MMPs activity associated with increase in glomerular MMP-1 by immunohistochemistry. These MMPs changes were occurring intracellulary and not extracellulary. ${ }^{64)}$ Examination of the literature reveals several studies that have reported predominantly intracellular location of MMPs or TIMPs in experimental CKD. ${ }^{41,43,53,65,66)}$

\section{MMPS AND THEIR INHIBITORS IN HUMAN KIDNEY DISEASE}

There are limited number of reports of MMPs/TIMPs expression in human kidney disease. Human kidney biopsies with diabetic nephropathy showed expression of MMP-3, and TIMP1 mRNA levels were associated with matrix accumulation characteristic of progressive diabetic nephropathy. ${ }^{67)}$ In addition, MMP-2 mRNA expression was found to be low in glomeruli of non insulin dependant diabetic patients, suggesting that MMPs may play a pivotal role in matrix accumulation in diabetic nephropathy. ${ }^{68)}$ In situ hybridization on renal biopsy specimens from patients with IgA nephropathy showed MMP-3, and TIMP-1 mRNA expression in glomerular cells, and tubular epithelial cells, was upregulated in association with progression of the disease. ${ }^{8)}$ However MMP-9 was significantly increased in mesangial proliferative glomeruli and interstitial vascular walls of $\operatorname{IgA}$ nephropathy patients, but markedly decreased in sclerotic glomeruli, and not changed in the tubules. ${ }^{69)}$ It was reported that, in human adult polycystic kidney disease (ADPKD), increased levels of MMP-2 and -9 and stromelysin were localized to tubular cells, with the greatest amounts in the collecting duct epithelia. ${ }^{70)}$ The expression of glomerular and interstitial MMP-2, -3, -9 and TIMP-1 is increased in active ANCA-associated glomerulonephritis and was shown to correlate with inflammatory activity. ${ }^{71)}$ MMP-9 was thought to play important role in abnormal mesangial proliferative changes in human GN; glomerular MMP9 immunostaining increased in parallel with levels of mesangial proliferative changes in IgA nephritis, Henoch-Shönlein nephritis, non-IgA mesangial proliferative GN and in lupus nephritis GN. Also increased gelatinolytic glomerular MMP-9 activ- 
ity was observed in nephritic glomeruli with $\operatorname{IgA}$ nephritis, lupus nephritis and DN. ${ }^{72)}$ In chronic allograft nephropathy (CAN), elevated serum proMMP2 and proMMP-3 levels were shown to reflect the changes in glomerular and interstitial extracellular matrix in chronic transplant nephropathy, suggesting that they could play a role in the pathogenesis of CAN. ${ }^{73-75)}$ Also in acute renal allograft rejection increased serum level of proMMP-1 was found, which could be a reflection of the stimulation induced by a number of inflammatory cytokines produced in such a process. ${ }^{73,74)}$ TIMP-1 and TIMP2 genes were expressed in normal glomeruli, their level of expression was increased in glomerulosclerosis associated with renal carcinoma, suggesting that expression of these inhibitors may correlate with the development of kidney scarring. ${ }^{75)} \mathrm{We}$ did examine MMP-1, 2, 3, and 9 in chronic allograft dysfunction using immunohistochemistry and they were significantly reduced in comparison to elevated TIMP-2, and -3 (unpublished data).

\section{CONCLUSION}

The involvement of the MMPs/TIMPs system in the kidney is so extensive starting from development, and has obvious role in progressive glomeuloscelosis and tubulo-interstitial fibrosis. However many interacting factors are also involved in the kidney ECM turnover, and there is no consistency in the literature regarding MMPs/TIMPs expression in various progressive CKD pathology. There are limitations of drawing conclusions based solely on mRNA and antigen measurements with such a complex pro enzyme/natural inhibitor system being in place. Hence the exact contribution of elevated TIMPs, MMPs activity in glomerulosclerosis, and tubulo-interstitial scarring remains unclear. Subsequently the development of MMPs induction/antiTIMPs strategies to combat renal scarring may at best prove ineffective and at worst accelerate glomerulosclerosis.

\section{REFERENCES}

1) Schnaper, H. W., Hayashida, T., Hubchak, S. C., Poncelet, A. C. (2003) TGF-beta signal transduction and mesangial cell fibrogenesis. Am. J. Physiol. Renal. Physiol., 284, F243-F252.

2) Liu, Y. (2006) Renal fibrosis: new insights into the pathogenesis and therapeutics. Kidney Int., 69, 213217.

3) Lenz, O., Elliot, S. J. and Stetler-Stevenson, W. G. (2000) Matrix metalloproteinases in renal development and disease. J. Am. Soc. Nephrol., 11, 574581.

4) Arthur, M. J. (1998) Fibrosis and altered matrix degradation. Digestion, 59, 376-380.

5) McDougall, S., et al. (2006) Fibroblast migration and collagen deposition during dermal wound healing: mathematical modelling and clinical implications. Philos. Transact. A, Math. Phys. Eng. Sci., 364, 1385-1405.

6) Jones, C. (1996) Matrix regulation in renal disease. Nephrology (Carlton), 2, 13-23.

7) Berkholtz, C. B., et al. (2006) Distribution of extracellular matrix proteins type I collagen, type IV collagen, fibronectin, and laminin in mouse folliculogenesis. Histochem. Cell Biol., 126, pp. 583-592.

8) Miyazaki, M., et al. (2003) Regulation of renal extracellular matrix metabolism. Contrib. Nephrol., 139, 141-155.

9) Mulloy, B. and Rider, C. C. (2006) Cytokines and proteoglycans: an introductory overview. Biochem. Soc. Trans., 34(Pt3), 409-413.

10) Bosman, F. T. and Stamenkovic, I. (2003) Functional structure and composition of the extracellular matrix. J. Pathol., 200, 423-428.

11) Brown, B., et al. (2006) The basement membrane component of biologic scaffolds derived from extracellular matrix. Tissue Eng., 12, 519-526.

12) Mott, J. D. and Werb, Z. (2004) Regulation of matrix biology by matrix metalloproteinases. Curr. Opin. Cell Biol., 16, 558-564.

13) Egeblad, M. and Werb, Z. (2002) New functions for the matrix metalloproteinases in cancer progression. Nat. Rev. Cancer, 2, 161-174.

14) Overall, C. M. (2001) Matrix metalloproteinase substrate binding domains, modules and exosites. Overview and experimental strategies. Methods Mol. Biol., 151, 79-120.

15) Sternlicht, M. D. and Werb, Z. (2001) How matrix metalloproteinases regulate cell behavior. Annu. Rev. Cell Dev. Biol., 17, 463-516.

16) Knauper, V., et al. (1997) The role of the C-terminal domain of human collagenase-3 (MMP-13) in the activation of procollagenase-3, substrate specificity, and tissue inhibitor of metalloproteinase interaction. J. Biol. Chem., 272, 7608-7616.

17) Knauper, V., et al. (1997) Activation of progelatinase B (proMMP-9) by active collagenase-3 (MMP13). Eur. J. Biochem., 248, 369-373.

18) Kojima, S., et al. (2000) Membrane-type 6 matrix 
metalloproteinase (MT6-MMP, MMP-25) is the second glycosyl-phosphatidyl inositol (GPI)-anchored MMP. FEBS Lett., 480, 142-146.

19) Catania, J. M., Chen, G. and Parrish, A. R. (2007) Role of matrix metalloproteinases in renal pathophysiologies. Am. J. Physiol. Renal. Physiol., 292, F905-F911.

20) Murphy, G., et al. (1999) Mechanisms for pro matrix metalloproteinase activation. APMIS, 107, 38-44.

21) Morrison, C. J. and Overall, C. M. (2006) TIMPindependence of MMP-2 activation by MT2-MMP is determined by contributions of both the MT2MMP catalyic and hemopexin C domains. J. Biol. Chem., 281, pp. 26528-26539.

22) Kwak, H. J., et al. (2006) Transforming growth factor-beta1 induces tissue inhibitor of metalloproteinase-1 expression via activation of extracellular signal-regulated kinase and Sp1 in human fibrosarcoma cells. Mol. Cancer Res., 4, 209-220.

23) Baricos, W. H., et al. (1995) ECM degradation by cultured human mesangial cells is mediated by a PA/plasmin/MMP-2 cascade. Kidney Int., 47, 10391047.

24) Chegini, N. (1997) The role of growth factors in peritoneal healing: transforming growth factor beta (TGF-beta). Eur. J. Surg. Suppl., (577), 17-23.

25) Naveau, A., et al. (2006) Phenotypic study of human gingival fibroblasts labeled with superparamagnetic anionic nanoparticles. J. Periodontol., 77, 238-247.

26) Seeland, U., et al. (2002) Myocardial fibrosis in transforming growth factor-beta(1) (TGF-beta(1)) transgenic mice is associated with inhibition of interstitial collagenase. Eur. J. Clin. Invest., 32, 295303.

27) Kitamura, M., et al. (1995) Transfer of a mutated gene encoding active transforming growth factorbeta 1 suppresses mitogenesis and IL-1 response in the glomerulus. Kidney Int., 48, 1747-1757.

28) Baker, A. H., Edwards, D. R. and Murphy, G. (2002) Metalloproteinase inhibitors: biological actions and therapeutic opportunities. J. Cell Sci., 115(Pt19), 3719-3727.

29) Gomis-Ruth, F. X., et al. (1997) Mechanism of inhibition of the human matrix metalloproteinase stromelysin-1 by TIMP-1. Nature, 389, 77-81.

30) Yu, W. H., et al. (2000) TIMP-3 binds to sulfated glycosaminoglycans of the extracellular matrix. $J$. Biol. Chem., 275, 31226-31232.

31) Orellana, S. A. and Avner, E. D. (1998) Cell and molecular biology of kidney development. Semin. Nephrol., 18, 233-243.

32) Ota, K., et al. (1998) Cloning of murine membrane- type-1-matrix metalloproteinase (MT-1-MMP) and its metanephric developmental regulation with respect to MMP-2 and its inhibitor. Kidney Int., 54, 131-142.

33) Barasch, J., et al. (1999) Tissue inhibitor of metalloproteinase-2 stimulates mesenchymal growth and regulates epithelial branching during morphogenesis of the rat metanephros. J. Clin. Invest., 103, 1299-1307.

34) Lelongt, B., et al. (1997) Matrix metalloproteinases MMP2 and MMP9 are produced in early stages of kidney morphogenesis but only MMP9 is required for renal organogenesis in vitro. J. Cell Biol., 136, 1363-1373.

35) Sakurai, H. and Nigam, S. K. (1997) Transforming growth factor-beta selectively inhibits branching morphogenesis but not tubulogenesis. Am. J. Physiol., 272(1Pt2), F139-F146.

36) Johnson, T. S., et al. (2002) Matrix metalloproteinases and their inhibitions in experimental renal scarring. Exp. Nephrol., 10, 182-195.

37) Yang, J., et al. (2002) Disruption of tissue-type plasminogen activator gene in mice reduces renal interstitial fibrosis in obstructive nephropathy. J. Clin. Invest., 110, 1525-1538.

38) Singhal, P. C., Sagar, S. and Garg, P. (1996) Simulated glomerular pressure modulates mesangial cell $72 \mathrm{kDa}$ metalloproteinase activity. Connect. Tissue Res., 33, 257-263.

39) Sagar, S., et al. (1994) Morphine modulates 72kDa matrix metalloproteinase. Am. J. Physiol., 267(4Pt2), F654-F659.

40) Reckelhoff, J. F. and Baylis, C. (1993) Glomerular metalloprotease activity in the aging rat kidney: inverse correlation with injury. J. Am. Soc. Nephrol., 3, 1835-1838.

41) Gonzalez-Avila, G., et al. (1998) Changes in matrix metalloproteinases during the evolution of interstitial renal fibrosis in a rat experimental model. Pathobiology, 66, 196-204.

42) Schafer, L., et al. (1995) Role of proteinases in renal hypertrophy and matrix accumulation. Nephrol. Dial. Transplant., 10, 801-807.

43) Nazneen, A., et al. (2002) Possible role of Ets-1 and MMP-1 in matrix remodeling in experimental cisplatin nephropathy. Med. Electron. Microsc., 35, 242-247.

44) Marti, H. P. (2000) Role of matrix metalloproteinases in the progression of renal lesions. Presse Med., 29, 811-817.

45) Salo, T., et al. (1991) Transforming growth factorbeta 1 up-regulates type IV collagenase expression in cultured human keratinocytes. J. Biol. Chem., 
266, 11436-11441.

46) Martin, J., et al. (1994) Identification and independent regulation of human mesangial cell metalloproteinases. Kidney Int., 46, 877-885.

47) Jain, S., Bicknell, G. R. and Nicholson, M. L. (2000) Molecular changes in extracellular matrix turnover after renal ischaemia-reperfusion injury. Br. J. Surg., 87, 1188-1192.

48) Dong, F. Q., et al. (2004) [Expression of matrix metalloproteinase-2 and-9 in kidney of diabetic rats]. Zhejiang Da Xue Xие Bao Yi Xue Ban, 33, 245-249.

49) Uchio, K., et al. (2000) Decreased matrix metalloproteinase activity in the kidneys of hereditary nephrotic mice (ICGN strain). Nephron, 86, 145151.

50) Koide, H., et al. (1997) Effect of unilateral nephrectomy on gene expression of metalloproteinases and their inhibitors. Nephron, 75, 479-483.

51) Schaefer, L., et al. (1997) Differential regulation of glomerular gelatinase B (MMP-9) and tissue inhibitor of metalloproteinase-1 (TIMP-1) in obese Zucker rats. Diabetologia, 40, 1035-1043.

52) McMillan, J. I., et al. (1996) Characterization of a glomerular epithelial cell metalloproteinase as matrix metalloproteinase-9 with enhanced expression in a model of membranous nephropathy. J. Clin. Invest., 97, 1094-1101.

53) Chromek, M., et al. (2003) Matrix metalloproteinase- 9 and tissue inhibitor of metalloproteinases1 in acute pyelonephritis and renal scarring. Pediatr. Res., 53, 698-705.

54) Eddy, A. A. (1996) Molecular insights into renal interstitial fibrosis. J. Am. Soc. Nephrol., 7, 24952508.

55) Eddy, A. A. (1996) Interstitial inflammation and fibrosis in rats with diet-induced hypercholesterolemia. Kidney Int., 50, 1139-1149.

56) Ishidoya, S., et al. (1996) Delayed treatment with enalapril halts tubulointerstitial fibrosis in rats with obstructive nephropathy. Kidney Int., 49, 11101119.

57) Sharma, A. K., et al. (1995) Altered expression of matrix metalloproteinase-2, TIMP, and TIMP-2 in obstructive nephropathy. J. Lab. Clin. Med., 125, 754-761.

58) Tang, W. W., et al. (1994) Extracellular matrix accumulation in immune-mediated tubulointerstitial injury. Kidney Int., 45, 1077-1084.

59) Nakamura, T., et al. (1995) Effect of a specific endothelin A receptor antagonist on murine lupus nephritis. Kidney Int., 47, 481-489.

60) Schaefer, L., et al. (1996) Tubular gelatinase A
(MMP-2) and its tissue inhibitors in polycystic kidney disease in the Han: SPRD rat. Kidney Int., 49, 75-81.

61) Nakamura, T., et al. (2000) Elevation of serum levels of metalloproteinase-1, tissue inhibitor of metalloproteinase-1 and type IV collagen, and plasma levels of metalloproteinase-9 in polycystic kidney disease. Am. J. Nephrol., 20, 32-36.

62) Duymelinck, C., et al. (1998) Inhibition of the matrix metalloproteinase system in a rat model of chronic cyclosporine nephropathy. Kidney Int., 54, 804-818.

63) Eddy, A. A., et al. (2000) Interstitial fibrosis in mice with overload proteinuria: deficiency of TIMP-1 is not protective. Kidney Int., 58, 618-628.

64) Ahmed, A. K., et al. (2007) Localization of matrix metalloproteinases and their inhibitors in experimental progressive kidney scarring. Kidney Int., 71, 755-763.

65) Kallakury, B. V., et al. (2001) Increased expression of matrix metalloproteinases 2 and 9 and tissue inhibitors of metalloproteinases 1 and 2 correlate with poor prognostic variables in renal cell carcinoma. Clin. Cancer Res., 7, 3113-3119.

66) Miyata, Y., et al. (2004) Expression of metalloproteinase-2, metalloproteinase-9, and tissue inhibitor of metalloproteinase- 1 in transitional cell carcinoma of upper urinary tract: correlation with tumor stage and survival. Urology, 63, 602-608.

67) Suzuki, D., et al. (1997) In situ hybridization studies of matrix metalloproteinase-3, tissue inhibitor of metalloproteinase-1 and type IV collagen in diabetic nephropathy. Kidney Int., 52, 111-119.

68) Del Prete, D., et al. (1997) Down-regulation of glomerular matrix metalloproteinase-2 gene in human NIDDM. Diabetologia, 40, 1449-1454.

69) Wang, J., et al. (2002) Expression of matrix metalloproteinase-9 and tissue inhibitor of metalloproteinase-1 in IgA nephropathy. Zhonghua Nei Ke Za Zhi, 41, 75-78.

70) Norman, J. T., et al. (1995) Matrix metalloproteinases and tissue inhibitor of matrix metalloproteinases expression by tubular epithelia and interstitial fibroblasts in the normal kidney and in fibrosis. Exp. Nephrol., 3, 88-89.

71) Sanders, J. S., et al. (2004) Renal expression of matrix metalloproteinases in human ANCA-associated glomerulonephritis. Nephrol. Dial. Transplant., 19, 1412-1419.

72) Urushihara, M., et al. (2002) Glomerular distribution and gelatinolytic activity of matrix metalloproteinases in human glomerulonephritis. Nephrol. Dial. Transplant., 17, 1189-1196. 
73) Rodrigo, E., et al. (2000) Changes in serum concentrations of matrix metalloproteinases in kidney transplantation. Transplant. Proc., 32, 517-518.

74) Rodrigo, E., et al. (2000) Circulating levels of matrix metalloproteinases MMP-3 and MMP-2 in renal transplant recipients with chronic transplant nephropathy. Nephrol. Dial. Transplant., 15, 20412045.

75) Carome, M. A., et al. (1993) Human glomeruli express TIMP-1 mRNA and TIMP-2 protein and mRNA. Am. J. Physiol., 264(6Pt2), F923-F929. 